\title{
Vaccination boosts adult immunity to varicella zoster virus
}

Background and epidemiology:

The recently published results of the large, randomized, placebocontrolled double-blind trial of the Shingles Prevention Study ${ }^{1}$ provide compelling evidence of the effectiveness of an investigational, highly potent vaccine against the varicella zoster virus in reducing the risk and severity of zoster (shingles) and postherpetic neuralgia among healthy adults aged 60 years or older. The investigational vaccine boosts cell-mediated immunity and is at least 14 times more potent than the vaccines to prevent varicella that are currently available.

After a median follow-up of 3 years, the investigators found that use of the zoster vaccine significantly reduced the incidence of shingles by $51 \%$ (5.4 v. 11.1 cases per 1000 person-years, $95 \%$ confidence interval $[\mathrm{CI}]$ $44 \%-58 \%)$ and post-herpetic neuralgia ( $0.46 \mathrm{v} .1 .38$ cases per 1000 person-years). Of the subjects who did develop shingles, those in the vaccinated group reported significantly less pain and discomfort (a $61 \%$ lower burden of disease, $95 \%$ CI 51\%-69\%) than those in the placebo group. However, during the first 42 days after vaccination the vaccine group had a significantly greater risk of a serious adverse event (1.9\% v. $1.3 \%)$ and experienced more adverse events at the injection site $(48.3 \%$ v. $16.6 \%$, $p$ $<0.05)$ than the placebo group.

In most cases, shingles (herpes zoster) is a reactivation of a childhood case of chickenpox (infection with the wild-type herpes varicella-zoster virus). At present, by age 15 about $95 \%$ of Canadians have had chickenpox. (This percentage is dropping, however, as parents take advantage of the recently available varicella vaccines, recommended as part of routine childhood immunization.) After infection, the varicella zoster virus lies dormant in the sensory ganglia of the cranial nerves and the ganglia of the spinal dorsal root. After a period of latency that can extend as long as decades, the virus may become reactivated and cause shingles.

Because the reactivation typically begins in the ganglia and travels along the peripheral nerves, patients often present with pain before the telltale dermatomal rash has appeared. The viral reactivation can also result in postherpetic neuralgia: about $40 \%$ of patients with shingles who do not take an antiviral therapy (and about $20 \%$ of those who do) will experience postherpetic neuralgia around 6 months after diagnosis.

The risk of herpes zoster increases with age. According to health care utilization data for 1979-1997, average annual consultation rates for herpes zoster in Canada ranged from 64 per 100000 children $0-4$ years of age to 812 per 100000 people aged 65 years and older. The average length of hospital stay for the latter age group was 20 days, which attests to the burden of this illness in older patients. ${ }^{2}$

Clinical management: Although visual diagnosis of varicella zoster infection is usually straightforward, the virus can be isolated from the vesicular skin lesions of either varicella or zoster, especially in the first day or 2 after onset of the rash. Other diagnostic approaches include direct immunofluorescence and polymerase chain reaction assay.

Although antiviral therapy is not indicated for chickenpox in immunocompetent children, for adults with chickenpox it should be initiated within 24 hours of the onset of rash. For shingles, it should be initiated within 72 hours of rash onset or earlier (early treatment leads to better outcomes). First-line therapy in the normal, immunocompetent patient with shingles is a 7-day course of either famciclovir (500 $\mathrm{mg}$, twice daily) or valacyclovir (1 g, twice daily). ${ }^{3}$ Concomitant analgesics, such as NSAIDs or acetaminophen with codeine, may also be indicated. There is growing evidence that aggressive and effective relief of acute neuropathic pain may reduce the risk of chronic pain.

Prevention: Increasing uptake of the currently available varicella vaccine to prevent childhood chickenpox may paradoxically increase the incidence of herpes zoster among older generations who have had natural varicella and harbour the virus. Several recent studies have suggested that intermittent exposure to varicella boosts cell-mediated immunity and protects against zoster. As fewer and fewer children experience chickenpox, we may see the incidence of zoster increase in our aging population. The Shingles Prevention Study results have inspired some individuals to recommend fast-tracking regulatory approval of the new potent zoster vaccine, with the proviso that the vaccinated be followed closely to better understand the risk and types of adverse reactions.

\section{Erica Weir \\ CMAF}

\section{References}

1. Oxman M, Levin M, Johnson G, Schmader K, Straus M, Gelb L, et al. A vaccine to prevent herpes zoster and postherpetic neuralgia in older adults. NE7M 2005;352(22):2271-84.

2. Brisson M, Edmunds W, Law B, Gay N, Walld R, Brownell M. Epidemiology of varicella zoster virus infection in Canada and the United Kingdom. Epidemiol Infect 2001;127(2):305-14.

3. Rosser W, Pennie R, Pilla $\mathrm{N}$ and the Anti-infective Review Panel. Antiinfective guidelines for communityacquired infections. Toronto: MUMS (Medication Use Management Services) Guideline Clearinghouse; 2005. p. 50. 